\title{
Peptides corresponding to helices 5 and 6 of Bax can independently form large lipid pores
}

\author{
Ana J. García-Sáez ${ }^{1}$, Manuela Coraiola ${ }^{3}$, Mauro Dalla Serra ${ }^{3}$, Ismael Mingarro ${ }^{1}$, Peter Müller ${ }^{4}$ \\ and Jesús Salgado ${ }^{1,2}$ \\ 1 Department of Biochemistry and Molecular Biology, University of Valencia, Spain \\ 2 Institute of Molecular Science, University of Valencia, Spain \\ 3 ITC-CNR Institute of Biophysics, Trento, Italy \\ 4 Institut für Biologie/Biophysik, Humboldt-Universität zu Berlin, Germany
}

\author{
Keywords \\ amphipathic peptides; apoptosis; Bcl2 \\ proteins; membrane proteins; toroidal pores \\ Correspondence \\ J. Salgado, Instituto de Ciencia Molecular, \\ Universitat de València, Edificio de Institutos \\ de Paterna, Polígono la Coma s/n, 46980 \\ Paterna, Valencia, Spain \\ Fax: +349635443273 \\ Tel: +349635 43016 \\ E-mail: jesus.salgado@uv.es
}

(Received 14 October 2005, revised 23 December 2005, accepted 28 December 2005)

doi:10.1111/j.1742-4658.2006.05123.x
Proteins of the B-cell lymphoma protein $2(\mathrm{Bcl} 2)$ family are key regulators of the apoptotic cascade, controlling the release of apoptotic factors from the mitochondrial intermembrane space. A helical hairpin found in the core of water-soluble folds of these proteins has been reported to be the poreforming domain. Here we show that peptides including any of the two $\alpha$-helix fragments of the hairpin of $\mathrm{Bcl} 2$ associated protein $\mathrm{X}$ (Bax) can independently induce release of large labelled dextrans from synthetic lipid vesicles. The permeability promoted by these peptides is influenced by intrinsic monolayer curvature and accompanied by fast transbilayer redistribution of lipids, supporting a toroidal pore mechanism as in the case of the full-length protein. However, compared with the pores made by complete Bax, the pores made by the Bax peptides are smaller and do not need the concerted action of tBid. These data indicate that the sequences of both fragments of the hairpin contain the principal physicochemical requirements for pore formation, showing a parallel between the permeabilization mechanism of a complex regulated protein system, such as Bax, and the much simpler pore-forming antibiotic peptides.
Mitochondria provide a critical control point for the apoptotic route [1]. The intermembrane spaces of these organelles function as storage compartments for a number of pro-death proteins, such as cytochrome $c$, which are released into the cytoplasm as a consequence of apoptotic signals. The release of these apoptotic factors involves alteration of the mitochondrial outer membrane barrier, and is controlled by members of a large family of proteins known as B-cell lymphoma protein 2 ( $\mathrm{Bcl} 2)$ [2].

$\mathrm{Bc} 2$ associated protein $\mathrm{X}(\mathrm{Bax})$ is a prototype, prodeath member of the Bcl2 family [3]. Although this protein is normally found soluble in the cytoplasm, its apoptotic activation includes extensive structural reorganization, which facilitates targeting to, and insertion into, the mitochondrial outer membrane [4-6]. The latter process is promoted by other types of $\mathrm{Bcl} 2$ protein, exemplified by Bid, that share with the former only the BH3 domain [7-9]. Proteolitically activated Bid (tBid) may, in turn, activate Bax through $\mathrm{BH} 3$-dependent or BH3-independent interactions [10]. Once in the mitochondrial outer membrane, Bax forms oligomeric pores and facilitates the release of cytochrome $c[9,11]$.

In vitro, Bax can promote the release of fluorescent probes and cytochrome $c$ from large unilamellar vesicles (LUVs) [12]. The characteristics of this activity and the properties of ion channels measured in planar lipid bilayers [13] led Basañez and coworkers [14] to propose that Bax forms partially lipid pores. Thus, the permeabilizing activity of Bax depends on the curvature properties of the bilayer, being induced by nonlamellar lipids with positive intrinsic curvature, but inhibited by lipids with negative intrinsic curvature $[10,15]$. Other cell-death-related proteins, such as

\section{Abbreviations}

Bax, Bcl2 associated protein X; Bcl2, B-cell lymphoma protein 2; LUV, large unilamellar vesicle. 
colicins [16] and actinoporins [17,18], and helical antimicrobial peptides, such as magainin [19,20] and melittin [21], have also been reported to form partially lipid pores, often described as toroidal because of their assumed geometry. These structures are thought to be similar to purely lipid holes, such as those formed under a number of stress conditions [22], but with the wall of the pore made of both lipid and protein molecules. At the rim of the membrane hole, the two leaflets of the bilayer can make contact with each other, forming a membrane edge with positive monolayer curvature at a plane perpendicular to the bilayer and negative monolayer curvature in the plane of the membrane [17,20,23] (Fig. 1). The organization of the protein or peptide molecules in the pore is not yet known, but they can be assumed to always lie embedded in the polar surface of the membrane, with variable insertion into the hydrocarbon region [20]. It is assumed that a number of peptide (or protein)

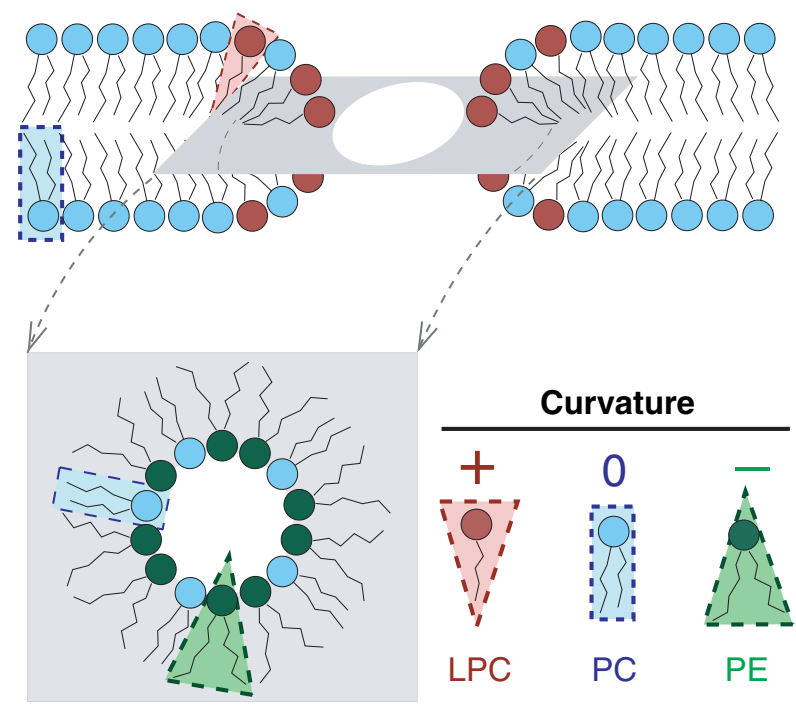

Fig. 1. Schematic representation of the idealized geometry of a lipid pore and the expected effect of spontaneous curvature of lipids. The rim of a lipid pore can be represented as a semi-toroidal surface at the point where the two monolayers fuse. This is characterized by strong curvature stress, caused by inefficient molecular packing, which causes high line tension and makes the pores energetically unfavourable. Because of the saddle-like geometry at the rim, both positive curvature, in a plane perpendicular to the membrane, and negative curvature, in the plane of the membrane, are present. In contrast with cylindrically shaped lipids, such as PtdCho (PC), lipids with anisotropic, cone-like shapes can improve packing at the rim and increase the stability of the pore. The latter is the case for lysolipids, such as lysoPtdCho (LPC), with positive intrinsic curvature, and the $H_{\|}$phase-inducing lipids, such as PtdEtn (PE), with negative intrinsic curvature. The positive curvature should have a dominant effect in most cases, as the negative curvature is expected to be important only for small pores [23]. molecules participate in the architecture of the supramolecular pore complex, although direct peptide-peptide interactions would in principle not be required.

The structure of water-soluble forms of a number of $\mathrm{Bcl} 2$ proteins is known (see, for example [24,25]). The most characteristic feature of this structure is a double-helix hairpin buried in the core of the proteins. This domain has the capacity to bind strongly to lipid membranes [26] and is regarded as being responsible for pore formation [27]. In line with this idea, we have shown recently that a peptide derived from the first helix of the hairpin of Bax can permeabilize model membranes, with characteristics that can be ascribed to the formation of partially lipid pores [28]. In this paper we describe the permeabilizing activity of a peptide derived from the second helix of the putative pore-forming domain of Bax. We show that peptides with the sequence of the first $(\alpha 5)$ and second $(\alpha 6)$ helices of the Bax hairpin (Fig. 2) can independently reproduce important characteristics of the activity of the full-length parent protein. In both cases, membrane pores are formed, which are able to export highmolecular-mass dextrans and are influenced by lipid spontaneous curvature. Pore formation is accompanied by lipid redistribution between the bilayer leaflets, supporting the model of a toroidal pore and in good agreement with the characteristics of the pore formed by Bax.

Although the individual peptide fragments do not exhibit the regulatory properties of the full-length parent protein, this work demonstrates the usefulness of short peptides as simple model systems in helping us to understand fundamental aspects of complex functional mechanisms.

\section{Results and discussion}

\section{Peptides of helices 5 and 6 from Bax are mainly $\alpha$-helical in lipid-mimetic environments}

The peptides used for this study (Fig. 2) are part of the characteristic hairpin that is taken as a signature domain of Bcl-2s and other structurally analogous pore-forming proteins. They contain the segments that in the full-length, water-soluble Bax make the fifth and sixth $\alpha$-helices of the protein structure [25]. However, when these fragments are taken out of their protein context and placed in a lipid environment, an $\alpha$-helical structure should not necessarily be assumed. To check if these peptides indeed arrange themselves into helical structures, we performed CD experiments in different lipid-mimetic media. 


\section{$\alpha 5 \quad \alpha 6$}
A
102
DGNFNWGRVVALFYFAS
B
Ac-KGRVVALFYFASKLVLKALSTKVPELIRTK-NH
C
Ac-DGNFNWGRVVALFYFASKLVLKALSTKVPELIRT- $\mathrm{NH}_{2}$
D

\author{
$\mathrm{Ac-ELIRTIMGWTLDFLRERLLVWIQD-} \mathrm{NH}_{2}$
}

Fig. 2. Part of the sequence of $B c l 2$ associated protein $X(B a x)$ and the peptides investigated in this work. The fragment from residues $102-$ 154 of mouse Bax is shown, which includes the $\alpha 5-\alpha 6$ hairpin (A), with residues found in $\alpha$-helix in the structure of the water-soluble form of the protein [25] highlighted in white over a dark grey background. Aligned are the sequence of the peptide Bax- $\alpha 5^{\mathrm{KK}}(\mathrm{B})$, studied previously [28], and the sequences of Bax- $\alpha 5$ (C) and Bax- $\alpha 6$ (D), studied here, with relevant residues highlighted: positively charged residues are coloured blue, negatively charged residues are coloured red, and residues substituted with respect to the wild-type sequence are printed over a light grey background.

As we can qualitatively appreciate in the CD spectra of Fig. 3, both peptides already display significant helicity in aqueous solvent (dotted lines). Under these conditions, values of $\approx 40 \%$ total helical structure, considering both regular and distorted $\alpha$-helix, are calculated in the case of Bax- $\alpha 5$ and $\approx 20 \%$ in the case of Bax- $\alpha 6$ (Table 1). The presence of even moderate amounts of SDS (Fig. 3A,C) or trifluoroethanol
(Fig. 3B,D) induces large increases in helical structure. Thus, $0.5 \mathrm{~mm}$ SDS is enough to induce more than $80 \% \alpha$-helix in Bax- $\alpha 5$ and about $50 \%$ in the case of Bax- $\alpha 6$. With $20 \%$ trifluoroethanol, Bax- $\alpha 5$ reaches almost complete helix induction $(\approx 90 \%)$, and $\approx 42 \%$ of Bax- $\alpha 6$ is found with helical conformation. Further increases of lipid-mimetic reagents, up to $4 \mathrm{~mm}$ SDS or $40 \%$ trifluoroethanol, induce helicity values up to
Fig. 3. $C D$ spectra of Bax- $\alpha 5$ and Bax- $\alpha 6$ in lipid-mimetic media. $C D$ spectra are shown for Bax- $\alpha 5(A, B)$ and Bax- $\alpha 6(C, D)$ in pure aqueous buffer (dotted lines) or in the presence of different concentrations of SDS (solid lines, A and C) or trifluoroethanol (solid lines, B and D). In all samples the peptide was at a concentration of $30 \mu \mathrm{M}$, and the aqueous buffer was $10 \mathrm{~mm}$ sodium phosphate, $\mathrm{pH}$ 7.0. The amounts of added trifluoroethanol (percentage) and SDS $\left(\mathrm{mM} \cdot \mathrm{L}^{-1}\right)$ are indicated on the graphs.
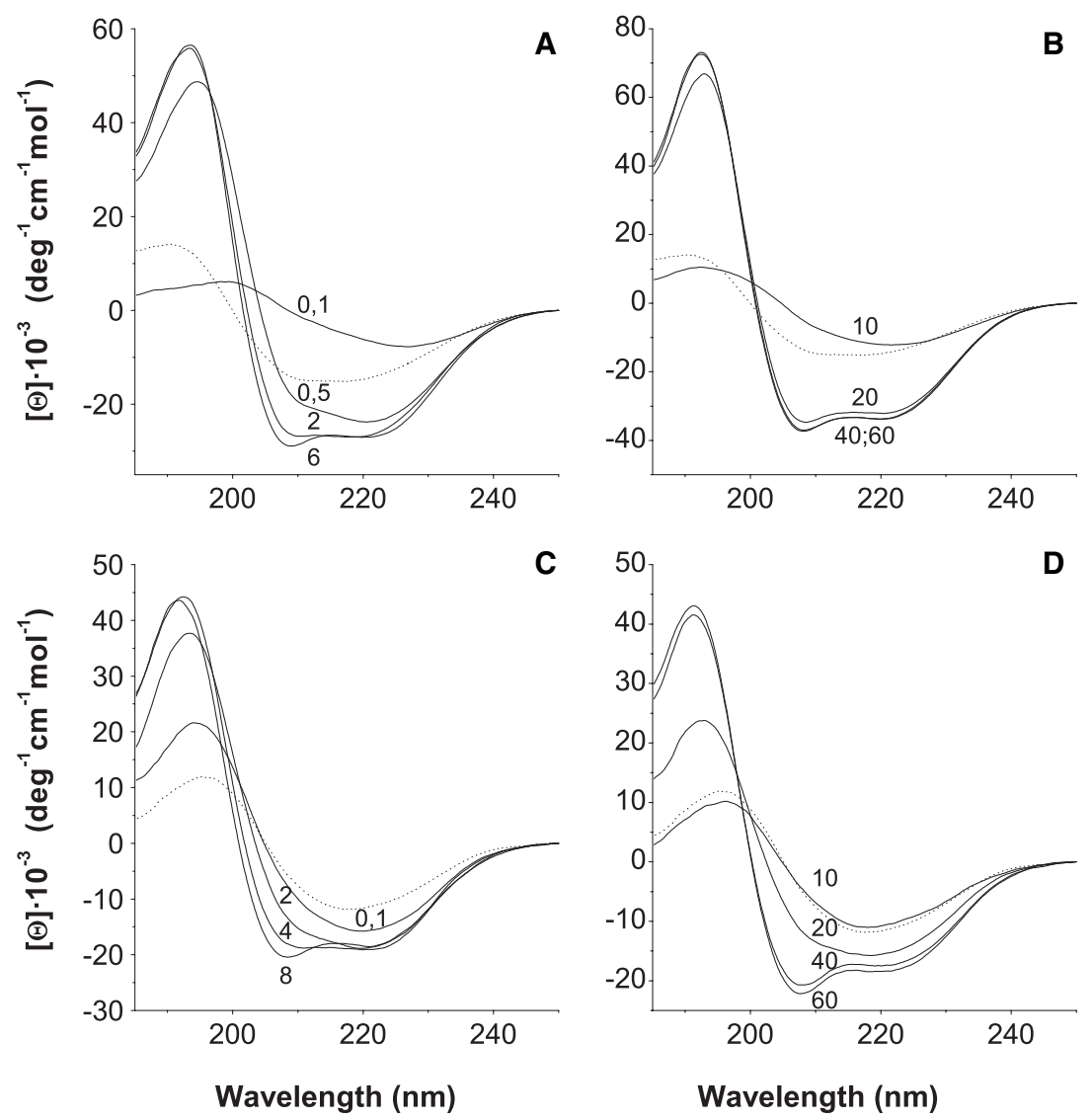
Table 1. Secondary structure of Bax- $\alpha 5$ and Bax- $\alpha 6$ peptides after analysis of the CD spectra. $H$, helix; $S$, strand; Trn, turn; Unrd, unordered; ( $r$ ), regular; (d), distorted, as defined by the standard output of CDPro. Mean is the average of the results obtained with CDSSTR, CONTIN/LL and SELCON3 [42,43], and SD is the standard deviation. All measurements were performed at $30 \mu \mathrm{m}$ concentration of the corresponding peptide. Buffer medium was $10 \mathrm{mM} \mathrm{NaH} \mathrm{PO}_{4}$ at $\mathrm{pH}$ 7. Trifluoroethanol (TFE) or SDS was added at the concentrations indicated, over 10 mM $\mathrm{NaH}_{2} \mathrm{PO}_{4}$.

\begin{tabular}{|c|c|c|c|c|c|c|c|c|c|c|c|c|c|}
\hline \multirow[b]{3}{*}{ Peptide } & \multirow[b]{3}{*}{ Medium } & \multicolumn{12}{|c|}{$\%$ Secondary structure } \\
\hline & & \multicolumn{2}{|l|}{$H(r)$} & \multicolumn{2}{|l|}{$H(d)$} & \multicolumn{2}{|l|}{$S(r)$} & \multicolumn{2}{|l|}{$S(d)$} & \multicolumn{2}{|l|}{$\operatorname{Trn}$} & \multicolumn{2}{|l|}{ Unrd } \\
\hline & & Mean & SD & Mean & SD & Mean & SD & Mean & SD & Mean & $\mathrm{SD}$ & Mean & SD \\
\hline \multirow[t]{5}{*}{ Bax- $-\alpha 5$} & Buffer & 23 & 6 & 15 & 2 & 10 & 2 & 7 & 1 & 19 & 4 & 26 & 3 \\
\hline & $20 \%$ TFE & 62 & 5 & 26 & 2 & 0 & 1 & 0 & 1 & 3 & 1 & 9 & 6 \\
\hline & $60 \%$ TFE & 61 & 5 & 27 & 2 & 0 & 1 & 0 & 2 & 1 & 1 & 11 & 5 \\
\hline & $0.5 \mathrm{~mm}$ SDS & 63 & 6 & 19 & 3 & 1 & 2 & 0 & 1 & 4 & 4 & 13 & 4 \\
\hline & $4 \mathrm{~mm}$ SDS & 61 & 4 & 25 & 3 & 0 & 1 & 0 & 1 & 4 & 2 & 10 & 6 \\
\hline \multirow[t]{5}{*}{ Bax- $\alpha 6$} & Buffer & 13 & 1 & 10 & 1 & 20 & 4 & 9 & 1 & 20 & 2 & 28 & 3 \\
\hline & $20 \%$ TFE & 27 & 4 & 15 & 0.2 & 8 & 1 & 6 & 0.2 & 18 & 2 & 26 & 2 \\
\hline & $60 \%$ TFE & 40 & 4 & 22 & 2 & 2 & 1 & 3 & 1 & 12 & 3 & 21 & 2 \\
\hline & $0.5 \mathrm{~mm}$ SDS & 33 & 3 & 16 & 0.2 & 8 & 1 & 5 & 0.3 & 15 & 2 & 23 & 3 \\
\hline & $4 \mathrm{~mm}$ SDS & 42 & 1 & 19 & 0.3 & 3 & 1 & 2 & 0.3 & 11 & 1 & 22 & 1 \\
\hline
\end{tabular}

$\approx 60 \%$ in Bax- $\alpha 6$ (Table 1). Helix induction takes place with a reduction in turn and unordered randomcoil structures, but especially at the expense of the $\beta$-strand structures, which are practically absent in the presence of the lipid-mimetic reagents (Table 1).

It is interesting to compare these results with our previous structural study of a different peptide that also included the sequence corresponding to $\alpha$-helix 5 of Bax (Fig. 2B,C) [28], although it was shorter by six residues at the $\mathrm{N}$-terminus and contained two extra, non-natural Lys residues, one at each end, compared with Bax- $\alpha 5$ studied here. For the sake of clarity, we will refer to the peptide studied previously as Bax$\alpha 5^{\mathrm{KK}}$. The observed structural consequence of the differences between Bax- $\alpha 5$ and $\mathrm{Bax}-\alpha 5^{\mathrm{KK}}$ is a larger intrinsic tendency of the former to fold into an $\alpha$-helix. Thus, for Bax- $\alpha 5$, this type of structure, represented by the sum of regular and distorted helices (Table 1), is the most abundant one, even in pure aqueous buffer, and it dominates in lipid-mimetic media. Bax- $\alpha 5^{\mathrm{KK}}$ has also been investigated by Fourier transform infraredattenuated total reflection in oriented lipid-bilayer samples using polarized light [28]. Two distinct types of $\alpha$-helix, oriented differently, were found in this case: one regular type of $\alpha$-helix was tilted with respect to the membrane plane, whereas one distorted helix was laying flat in the membrane. The tilted helical stretch probably corresponded to the more hydrophobic $\mathrm{N}$-terminal side of the peptide. This part has been extended in the case of Bax- $\alpha 5$, which may stabilize a longer $\mathrm{N}$-terminal helix and explain the increase in the activity of this peptide version with respect to Bax- $\alpha 5^{\mathrm{KK}}$ (see below).

\section{Helices 5 and 6 of Bax independently permeabilize vesicles, with activities affected by lipid spontaneous curvature}

The peptides encompassing helices 5 and 6 of Bax display a potent calcein-release activity in LUVs of different compositions. The dependence of vesicle poration on the concentration of the peptides typically yields sigmoidal curves, indicative of co-operativity, with inflexion points corresponding to peptide concentrations giving $50 \%$ of maximum activity $\left(\mathrm{IC}_{50}\right)$. With LUVs made of PtdCho at $6 \mu \mathrm{m}$ lipid concentration, $\mathrm{IC}_{50}$ values are in the nanomolar range for Bax- $\alpha 5$ (Fig. 4A, squares and continuous line), and about an order of magnitude higher for Bax- $\alpha 6$ (Fig. 4B). The process also depends on the lipid composition of the vesicles, as summarized in Table 2. For both Bax- $\alpha 5$ and Bax- $\alpha 6$, the activity values, expressed as $1 / \mathrm{IC}_{50}$, are larger in neutral lipid bilayers made of PtdCho than in the presence of negatively charged lipids such as PtdSer and cardiolipin. In the case of Bax- $\alpha 5$, which possess a net positive charge (Fig. 2), we would expect increased binding to negatively charged membranes, as observed previously for $\mathrm{Bax}-\alpha 5^{\mathrm{KK}}$ [28]. Thus, it appears that electrostatic forces exert a negative effect on the activity of these peptides, probably through increased binding which favours an inactive state with the peptide adsorbed at the membrane interface (but see below).

The poration activity of full-length Bax and Bax-like proteins is greatly influenced by the intrinsic monolayer curvature of the membrane lipids. The presence of lipids with a positive intrinsic curvature, such as 

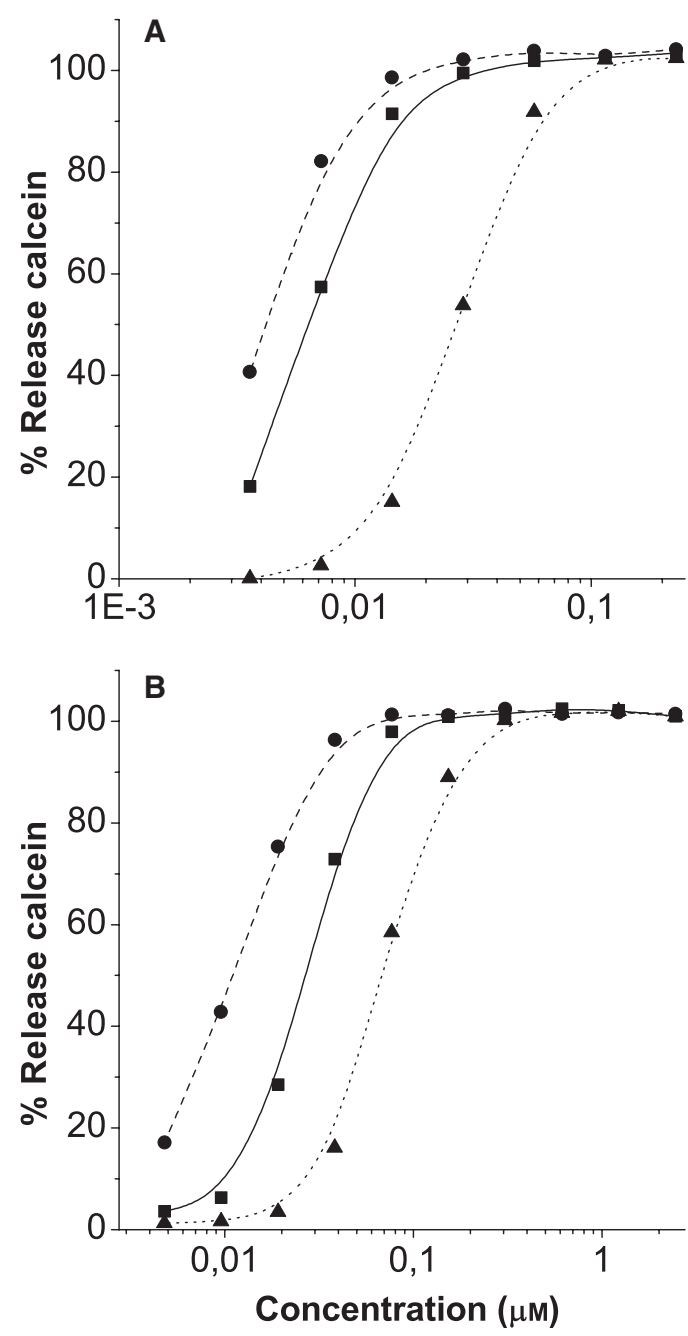

Fig. 4. Calcein release from LUVs induced by the Bax- $\alpha 5$ and Bax$\alpha 6$ peptides. The percentage calcein release calculated with eqn (1) is represented as a function of the concentration of added peptide. (A) shows the effect of Bax- $\alpha 5$, and (B) corresponds to Bax- $\alpha 6$. Vesicles were prepared with the following compositions: (squares) egg PtdCho; (circles) PtdCho:lysoPtdCho (90: 10); (triangles) PtdCho:PtdSer (50: 50). Total lipid composition was $\approx 6 \mu \mathrm{M}$.

lysophospholipids, enhances Bax activity, and the opposite is observed for lipids with a negative intrinsic curvature $[10,14,15]$. Similarly, small percentages of lysoPtdCho increased the activity of the previously investigated peptide variant $\mathrm{Bax}-\alpha 5^{\mathrm{KK}}$, although in contrast with that observed for the complete protein, the negatively curved lipid PtdEtn also produces a significant increase in peptide activity [28].

For the Bax- $\alpha 5$ and Bax- $\alpha 6$ peptides studied here, clear increases in calcein release from LUVs were obtained in the presence of moderate amounts (up to $10 \%$ ) of lysoPtdCho (Fig. 4A,B and Table 2). The effect of the nonlamellar lipid PtdEtn follows a
Table 2. Effect of the lipid composition of LUVs on the release of calcein exerted by Bax- $\alpha 5$ and Bax- $\alpha 6$. Lipid mixtures are reported on a molar basis. $1 / \mathrm{C}_{50}$ is defined as the inverse concentration of the particular peptide causing $50 \%$ of calcein release. Typical standard deviations of the reported $1 / C_{50}$ values were $8-12 \%$. CL, cardiolipin.

\begin{tabular}{lcc}
\hline & \multicolumn{2}{c}{ Activity, $1 / \mathrm{C}_{50}\left(\mu \mathrm{M}^{-1}\right)$} \\
\cline { 2 - 3 } Lipid composition & Bax- $\alpha 5$ & Bax- $\alpha 6$ \\
\hline PtdCho & 158.7 & 35.2 \\
PtdCho:PtdSer $(75: 25)$ & 59.3 & 15.8 \\
PtdCho:PtdSer $(50: 50)$ & 37.6 & 13.8 \\
PtdCho:CL $(90: 10)$ & 35.3 & 5.7 \\
PtdCho:lysoPtdCho $(95: 5)$ & 215.1 & 53.1 \\
PtdCho:lysoPtdCho $(90: 10)$ & 238.1 & 69.4 \\
PtdCho:PtdEtn $(75: 25)$ & 198.0 & 28.3 \\
PtdCho:PtdEtn $(50: 50)$ & 175.4 & 16.3 \\
\hline
\end{tabular}

different trend for the two peptides, as summarized in Table 2. Thus, in the case of Bax- $\alpha 5$, the behaviour is similar to that reported for the Lys-flanking variant $\mathrm{Bax}-\alpha 5^{\mathrm{KK}}$, with small, but consistent, increases in activity in LUVs containing the nonlamellar lipid. However, the activity of Bax- $\alpha 6$ is reduced to about half of the baseline value in the presence of up to $50 \%$ PtdEtn. We assume a comparable binding of the peptides to membranes containing lysoPtdCho and PtdEtn lipids, with respect to pure PtdCho vesicles, as this has been shown for melittin, a peptide displaying properties and a mechanism similar to our Bax peptides [29].

The effects of spontaneous lipid curvature in the activity of pore-forming peptides are normally used as evidence in favour of mixed lipid/peptide toroidal pores, as opposed to barrel-stave pores [16,30]. The basis of this reasoning is that anisotropic inclusions are expected to relieve the curvature stress that exists at the membrane edge of the rim of toroidal pores, caused by monolayer fusion (Fig. 1). In the case of purely lipid pores at least, it has been shown experimentally, and modelled theoretically, that cone-shaped inclusions (with positive spontaneous curvature), such as detergent molecules, stabilize the pore by reducing the line tension of the pore rim, whereas inclusions with negative spontaneous curvature (inverted coneshaped) reduce the pore life time by increasing the line tension [31,32]. However, because of the complex, saddle-like geometry within the rim of toroidal pores, negative curvature stress is also expected at the membrane edge in a plane parallel to the bilayer (Fig. 1). In agreement with this interpretation, a detailed theoretical study predicts that saddle-like inclusions, characterized by both positive and negative intrinsic curvature, favour small pores, whereas wedge-like inclusions, with only positive curvature, stabilize larger 
pores [23]. Taking this into account, the increased poration activity observed for both Bax- $\alpha 5$ and Bax- $\alpha 6$ in the presence of lysoPtdCho indicates that these peptides act by forming toroidal pores. In addition, the complex heterogeneous effect of PtdEtn may indicate that these pores are of small to moderate size. A qualitative evaluation of this size is described in the next section.

We should point out that the effects of cardiolipin and PtdSer (see above), besides their negative charge, can be also interpreted in terms of their curvature properties, as these lipids are known to form $\mathrm{H}_{\mathrm{II}}$ phases under conditions of reduced interlipid electrostatic repulsion, as in the presence of $\mathrm{Ca}^{2+}$ or polylysine peptides [33]. Similar conditions could be achieved through the binding of polycationic Bax- $\alpha 5$ or Bax- $\alpha 6$ peptides, ending up with the imposition of a negative curvature strain. However, it is difficult to separate the influence of curvature from that of the net negative charge of the lipid, which may inhibit pore formation through alternative mechanisms. Thus, a stronger electrostatic interaction with negatively charged membranes may affect the transition of the peptide from an inactive surfacebound state to a pore-forming state $[28,34]$.

\section{Size of membrane holes made by Bax- $\alpha 5$ and Bax- $\alpha 6$ in LUVs}

Pores formed by $\mathrm{Bcl} 2$ proteins in the mitochondrial membrane must be large enough to allow passage of apoptotic factors stored in the intermembrane space, such as cytochrome $c$. It has been shown that Bax, assisted by $\mathrm{tBid}$, promotes the opening of large lipid pores, which do not impose a size-exclusion limit for labelled molecules of mass $\approx 0.4-70 \mathrm{kDa}$ [10]. Moreover, Bax accompanied by full-length Bid can promote the release of dextrans as large as $2000 \mathrm{kDa}$ from pure synthetic lipid vesicles [35]. We were thus interested in obtaining an estimate of the size of the pores formed by the $B a x-\alpha 5$ and $B a x-\alpha 6$ peptide fragments. We therefore studied the induced release from PtdCho LUVs of molecules larger than calcein $(\approx 0.6 \mathrm{kDa})$, such as $20 \mathrm{kDa}$ and $70 \mathrm{kDa}$ fluorescein-labelled dextrans (FD-20 and FD-70, respectively).

As occurred in the case of calcein, dextran release was markedly dependent on the lipid/peptide (L/P) molar ratio, but the leakage curves reached a plateau after a few minutes and complete release was not achieved for intermediate peptide concentrations (Fig. 5). This behaviour is common to other amphipathic lytic peptides, such as melittin and magainin, and it has been explained by an all-or-none mechanism of release because of the highly co-operative binding of the peptides to the lipid vesicles [34]. Both Bax- $\alpha 5$ and Bax- $\alpha 6$ were able to release high-molecular-mass dextrans, although, as observed for the experiments with calcein, Bax- $\alpha 6$ displayed a lower activity than Bax- $\alpha 5$, and the former needed about three times the concentration of the latter to promote a similar release of FD-20 or FD-70 (Fig. 5A-D). In contrast, a peptide
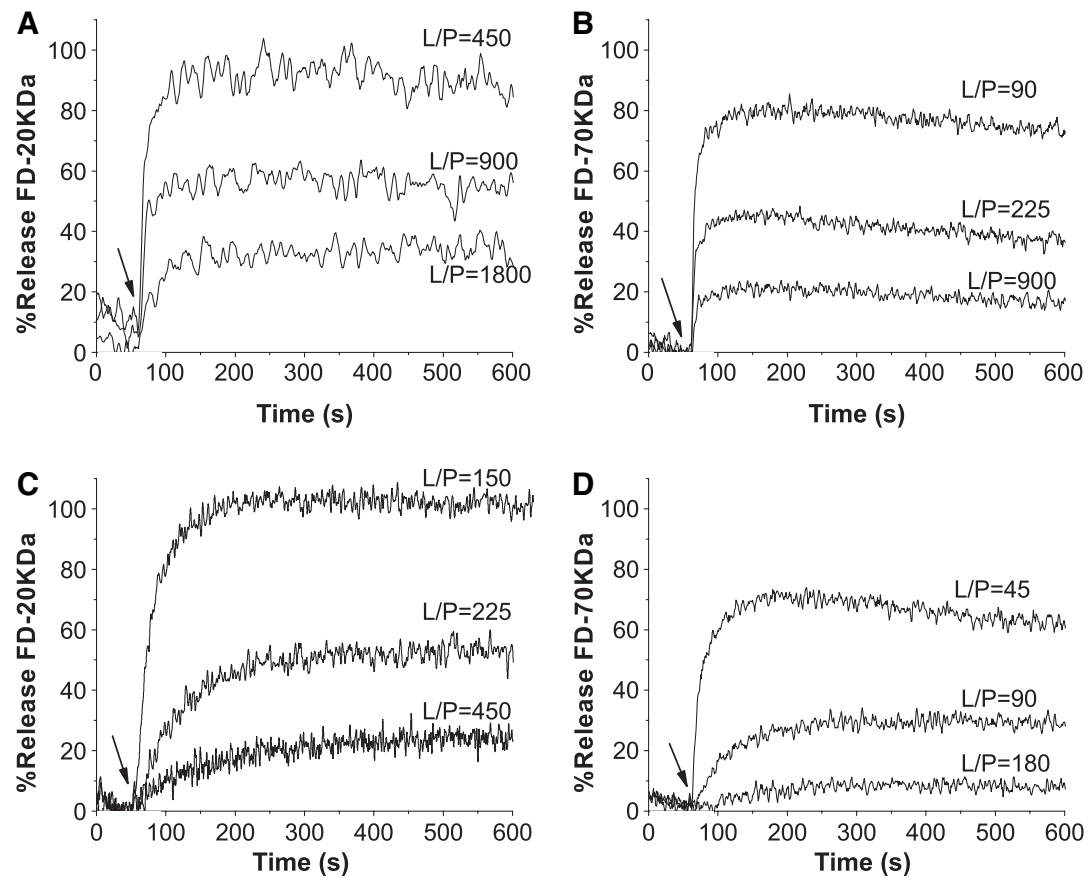

Fig. 5. Release of fluorescein-labelled dextrans from LUVs. A and B show the timedependent release of FD-20 and FD-70, respectively, from egg PtdCho LUVs as induced by Bax- $\alpha 5$ at the L/P molar ratios indicated on the graphs. $C$ and $D$ show similar experiments performed with Bax- $\alpha 6$ at the $L / P$ ratios indicated. Arrows indicate the time at which the corresponding peptides were added to the vesicle suspension. Lipid concentration was $45 \mu \mathrm{M}$. Release is expressed as percentage of the activity observed in the presence of $1 \mathrm{~mm}$ Triton $\mathrm{X}-100$, and calculated with eqn (1). 
corresponding to Bid- $\alpha 6$, which has been shown previously to be able to release calcein from PtdCho LUVs [28], assayed at $\mathrm{L} / \mathrm{P}$ molar ratios up to $\approx 5$, was unable to induce the release of FD-20 (not shown).

Before ascribing the increase in fluorescence to the effect of discrete large membrane pores, other peptidemediated effects, such as changes in the distribution volume through vesicle fusion or vesicle rupture through partial micellation or detergent-like mechanisms, as described for some antimicrobial peptides [36], must be considered. These possibilities were tested by measuring the changes in size of PtdCho LUVs after treatment with the peptides, using quasi-elastic light scattering. The results of a few representative experiments are collected in Table 3 . When the peptide Bax- $\alpha 5$ or Bax- $\alpha 6$ was added at concentrations that induced a substantial release of dextran, neither the size distribution of LUVs nor the intensity of the scattered light were affected, indicating that the observed increase in fluorescence in the experiments on dextran release is due to neither vesicle rupture nor vesicle fusion, but rather to peptide-induced membrane pores. As a positive control of vesicle solubilization, we added $1 \mathrm{~mm}$ Triton X-100, which is the detergent used to accomplish $100 \%$ release of dextrans. Triton X-100 dramatically decreased the intensity of the signal, which went below the detection limit of our instrument.

A size discrimination of the released molecules was also noticed, and lower $\mathrm{L} / \mathrm{P}$ ratios were needed to achieve a percentage release of FD-70 comparable to that of FD-20. This indicates that the size of the pores is dependent on the number of peptides bound per lipid vesicle. Thus, to a good approximation, for the range of $\mathrm{L} / \mathrm{P}$ values used in the experiments of Fig. 5, we can estimate the upper limit for the radius of pores made by Bax- $\alpha 5$ and Bax- $\alpha 6$ in PtdCho LUVs to be $5.8 \mathrm{~nm}$, corresponding to the hydrodynamic radius of

Table 3. Effects of Bax- $\alpha 5$, Bax- $-\alpha 6$ and Triton $X-100$ on the size of PtdCho LUVs. L/P, Lipid/peptide molar ratio. Lipid concentration was $25 \mu \mathrm{M}$. Triton X-100 concentration was $1 \mathrm{~mm}$. ND, not detectable.

\begin{tabular}{lrlll}
\hline Additive & L/P & $\begin{array}{l}\text { Average } \\
\text { size }(\mathrm{nm})\end{array}$ & Polydispersity & $\begin{array}{l}\text { Peak intensity } \\
(\mathrm{kc} / \mathrm{s})\end{array}$ \\
\hline None & 0 & $130.3 \pm 1.5$ & $0.11 \pm 0.05$ & $141.2 \pm 3.6$ \\
Bax- $\alpha 5$ & 500 & $129.6 \pm 1.2$ & $0.12 \pm 0.03$ & $147.0 \pm 3.5$ \\
& 250 & $131.4 \pm 1.2$ & $0.12 \pm 0.03$ & $149.0 \pm 4.4$ \\
& 50 & $140.3 \pm 0.9$ & $0.17 \pm 0.04$ & $159.6 \pm 2.9$ \\
Bax- $\alpha 6$ & 250 & $130.3 \pm 2.3$ & $0.11 \pm 0.02$ & $147.4 \pm 4.3$ \\
& 125 & $133.8 \pm 2.7$ & $0.16 \pm 0.02$ & $149.8 \pm 8.6$ \\
Triton X-100 & 50 & $141.3 \pm 4.0$ & $0.21 \pm 0.07$ & $149.9 \pm 9.3$ \\
& & ND & ND & $9.5 \pm 0.1$ \\
\hline
\end{tabular}

$70 \mathrm{kDa}$ dextrans [37]. This value is close to the smallsize condition of an ideal toroidal pore for which negative curvature at the pore rim is expected to be significant, corresponding to a radius close to half of the membrane thickness $(\approx 2 \mathrm{~nm})$ [23]. The above described heterogeneous effect of PtdEtn on the activity of Bax- $\alpha 5$ and Bax- $\alpha 6$ is in agreement with the moderate size of pores formed by these peptides. In contrast, pores made by the concerted action of Bax and tBid, at a $\mathrm{L} / \mathrm{P}$ ratio of 1000 [10], should have a bigger radius, as they were consistently inhibited by negatively curved lipids and exerted no size-exclusion limit for large dextrans. It is, however, interesting that size discrimination was observed for pores induced by the Bax $-\Delta \mathrm{C}+\mathrm{tBid}$ combination, in which Bax lacks the C-terminal putative transmembrane helix [10]. This may indicate a role for the C-terminal helix of Bax in the formation of large pores, although it may also be due to the influence of this domain on the efficiency of membrane insertion.

\section{The pore-forming activity of the Bax fragments is accompanied by lipid transbilayer redistribution}

As we have discussed, the formation of a lipid pore implies the existence of a membrane edge at the pore rim where lipids rearrange and tilt to shield their hydrocarbon chains from the aqueous environment (Fig. 1). This would effectively fuse the two leaflets of the membrane allowing fast transbilayer movement of lipids through lateral diffusion. Thus, fast lipid transbilayer redistribution is expected to accompany the formation of toroidal pores by peptides and proteins $[10,20,38]$. We have tested this possibility by using an assay developed by Muller et al. [39]. Briefly, it is based on the spectral changes observed on redistribution of the fluorescent PtdCho derivative pyrene labelled PtdCho (pyPtdCho), initially added to the external monolayer, to the inner leaflet of the membrane. The incorporation of pyPtdCho to LUVs composed of PtdCho was fast, and the ratio $I_{\mathrm{E}} / I_{\mathrm{M}}$ remained practically constant in the absence of peptides during a time course of $20 \mathrm{~min}$, indicating that the spontaneous transbilayer diffusion of the fluorescent analogue is negligible (not shown). The addition of Bax- $\alpha 5$ or Bax- $\alpha 6$ at nanomolar concentrations in the presence of $20 \mu \mathrm{M}$ lipid induced a rapid decrease in the $I_{\mathrm{E}} / I_{\mathrm{M}}$ ratio, indicating fast transbilayer lipid redistribution (Fig. 6A,B). As a negative control, the peptide Bid- $\alpha 6$, which forms pores probably of the barrel-stave type [28], produced no effect on the redistribution of pyPtdCho between the two monolayers when assayed at $\mathrm{L} / \mathrm{P}$ ratios as high as 10 (not shown). 

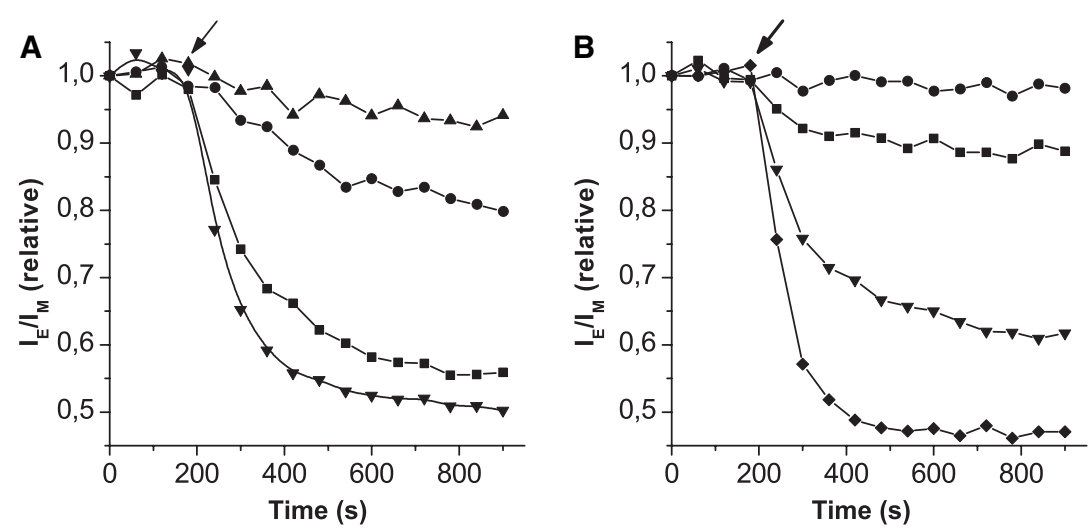

Fig. 6. Transbilayer redistribution of pyrene-labelled PtdCho induced by the peptide fragments Bax- $\alpha 5$ and Bax- $\alpha 6$ in LUVs. pyPtdCho was incorporated into PtdCho LUVs, and the time dependent decrease in $I_{E} / I_{M}$ induced at different molar L/P ratios was analysed as described in Experimental procedures: 200 (diamonds), 500 (triangles down), 1000 (squares), 2000 (circles), and 4000 (triangles up). The arrows indicate when peptides were added to a suspension of egg PtdCho vesicles at a $20 \mu \mathrm{M}$ lipid concentration. Represented $I_{E} / I_{M}$ ratios are referred to the $I_{E} / I_{M}$ value obtained in the absence of peptides, which is taken as 1 .

In agreement with the experiments on content release, we observed that the intrinsic transbilayer redistribution activity was higher in the case of Bax- $\alpha 5$ (Fig. 6A) than for Bax- $\alpha 6$ (Fig. 6B). In both cases, this activity was observed for concentrations of the same order of magnitude as that reported for full-length $\mathrm{Bax} / \mathrm{tBid}$ mixtures [10]. In addition, the process of lipid transbilayer redistribution is induced at $\mathrm{L} / \mathrm{P}$ ratios comparable to those needed for the release of dextrans, and also exhibited a similar time course, which indicates a mechanistic connection between the two observations. Both the release of high-molecular-mass dextrans and the increased lipid transbilayer diffusion on addition of peptides occurred without significantly affecting the size of the vesicles, excluding the possibility of a detergent-like action. Such behaviour would be expected if a mixed lipid/peptide pore with toroidal structure is being formed, and has also been reported for the concerted action of Bax and tBid [10].

\section{Concluding remarks}

In summary, peptides corresponding to natural sequences of Bax and encompassing individual helices of the characteristic $\alpha 5-\alpha 6$ hairpin domain can independently, and in the absence of tBid, reproduce the poration activity displayed in vitro by the concerted action of full-length Bax and tBid. Thus, our results suggest that Bax possesses, by itself, the intrinsic ability to form toroidal pores, and this ability is present in at least two helical fragments of its structure.

Both the $\alpha 5$ and $\alpha 6$ fragments are amphipathic and have positively charged Lys and/or Arg residues, similar to antibiotic peptides such as melittin and magai- nin, for which the formation of toroidal pores has also been described. We propose that the ability of Bax to form pores is inherently linked to the physicochemical properties of both fragments of the hairpin at least. Because the actions of the Bax fragments and the fulllength protein are observed at similar concentrations, a strong synergistic effect of the active fragments in the context of the whole protein is not expected. Rather, the complete structure may be important to achieve a larger pore size, as our data also indicate. Similarly, other important aspects of the function of Bax, such as the need to be activated through structural changes, the concerted action with other proteins such as tBid, and its oligomerization in the membrane, are certainly attributes of the whole protein that single fragments cannot reproduce. These complex aspects allow the function of Bax to be performed in a regulated manner, modulating the intrinsic poration activity of Bax fragment sequences to the level required for the correct functioning of the apoptotic route.

\section{Experimental procedures}

\section{Peptide synthesis and purification}

Two peptides containing the helices 5 (Bax- $\alpha 5$, Fig. 2C) and 6 (Bax- $\alpha 6$, Fig. 2D), corresponding to the structure of a soluble form of Bax, were synthesized chemically. Compared with a previous study [28], the version of Bax- $\alpha 5$ used for this work has been extended at the N-terminus and contains no extra flanking lysine residues (Fig. 2B,C). The only difference with respect to the natural sequences found in mouse Bax is the replacement of Cys126 with Ser in Bax- $\alpha 5$ to avoid dimerization via disulfide bridges. 
Solid-phase synthesis of the peptides was carried out as reported [28] in an Applied Biosystems (ABI, Foster City, CA, USA) 433A Peptide synthesizer using Fmoc chemistry and Tentagel S-RAM resin (Rapp Polymere, Tübingen, Germany; $0.24 \mathrm{mEq} / \mathrm{g}$ substitution) as a solid support. Peptides were purified using a $\mathrm{C} 18$ preparative reversed-phase column (Merck, Darmstadt, Germany) by HPLC, to a purity of $\approx 95 \%$, and their identity was confirmed by MS. All peptide concentrations were determined from UV spectra using a Jasco spectrophotometer (Jasco, Tokyo, Japan).

\section{Preparation and size measurement of vesicles}

All lipids used were from Avanti Polar Lipids (Alabaster, AL, USA). LUVs were prepared as described previously [40]. Lipids were dissolved in chloroform, mixed to the desired molar composition, and vacuum-dried to a thin film on the bottom of a round glass flask. Further drying was accomplished by $30 \mathrm{~min}$ in a strong vacuum. To prepare the fluorescein-bis(methyliminodiacetic acid) (calcein)-containing LUVs, lipids were resuspended to a concentration of $4 \mathrm{mg} \cdot \mathrm{mL}^{-1}$ in a solution containing $80 \mathrm{~mm}$ calcein (Sigma-Aldrich, St Louis, MO, USA), neutralized with $\mathrm{NaOH}$. After six cycles of freezing and thawing, they were passed 31 times through two stacked polycarbonate filters of 100-nm pore size, using a two-syringe extruder from Avestin (Ottawa, Ontario, Canada). To remove external nonencapsulated dye, LUVs were filtered on Sephadex G-50 (Sigma-Aldrich) mini-columns, previously equilibrated with $140 \mathrm{~mm} \mathrm{NaCl} / 20 \mathrm{~mm}$ Hepes/1 mm EDTA, pH 7 (buffer A). In the case of LUVs encapsulated with fluorescent dextrans (FD-20 or FD-70, from Sigma-Aldrich), the lipid films were resuspended to a concentration of $10 \mathrm{mg} \cdot \mathrm{mL}^{-1}$ in a $100 \mathrm{mg} \cdot \mathrm{mL}^{-1}$ solution of dextrans in buffer A. The vesicles were subjected to 20 cycles of freezing and thawing and extruded as described above, but using $200 \mathrm{~nm}$ polycarbonate filters. Nonencapsulated dextrans were removed by gel-filtration chromatography using an ÄKTA system (Amershan Pharmacia Biotech AB, Uppsala, Sweden) with a column $(35 \mathrm{~cm} \times 1.6 \mathrm{~cm})$ loaded with Sephacryl HS-500 (Amersham), equilibrated with buffer $\mathrm{A}$, at a flow rate of $0.5 \mathrm{~mL} \cdot \mathrm{min}^{-1}$. Fractions corresponding to the LUVs were pooled and used in the experiments on content release.

The size of the vesicles was measured by quasi-elastic light scattering at a fixed angle $\left(90^{\circ}\right)$ and room temperature, using a laser particle sizer (Malvern Z-sizer 3; Malvern, UK) upgraded with a $30 \mathrm{~mW}$ laser diode emitting at $675 \mathrm{~nm}$. Analysis of the autocorrelation function allows the estimation of the diffusion coefficient of the particles through Laplace inversion, from which the hydrodynamic radius is calculated using the cumulant method [41] and the StokesEinstein equation. The lipid concentration was estimated with the Phospholipids B kit (Wako Chemicals $\mathrm{GmbH}$, Neuss, Germany), following the supplier's protocol.

\section{Permeabilization of LUVs}

The permeabilizing activity of the peptides was assayed by measuring the release of the fluorescent probes calcein or fluorescein-labelled dextrans from LUVs. Release of the entrapped probe at self-quenching concentrations was monitored as an increase in the fluorescence intensity with time, due to dilution to nonquenching concentrations as the dye mixes with the external solution. Experiments on calcein release were performed using a 96-well microtitre plate filled with $100 \mu \mathrm{L}$ buffer A containing the desired amount of peptide. To avoid unspecific interactions of the peptides and vesicles with the plastic walls, the microplates were pretreated with a $0.1 \mathrm{mg} \cdot \mathrm{mL}^{-1}$ Prionex (Pentapharm, Basel, Switzerland) solution for $30 \mathrm{~min}$. All experiments were carried out at room temperature. After the addition of $100 \mu \mathrm{L}$ LUVs, at a final lipid concentration of $2-5 \mu \mathrm{g} \cdot \mathrm{mL}^{-1}$, the time course of calcein release was measured as the increase in fluorescence emission at $520 \mathrm{~nm}$ with the excitation set at $495 \mathrm{~nm}$, using a fluorescence microplate reader (FLUOstar; BMG Labtech $\mathrm{GmbH}$, Offenburg, Germany). The experiments measuring release of fluorescent dextrans were carried out using a cell of 1-cm path length in an LS-50B luminescence spectrometer (Perkin Elmer, Boston, MA, USA). The desired amount of peptide was added to the reaction mixture containing $1 \mathrm{~mL}$ buffer A and $100 \mu \mathrm{L}$ LUVs (at a final lipid concentration of $45 \mu \mathrm{M}$ ). The increase in fluorescence intensity at $520 \mathrm{~nm}$ (with excitation wavelength set at $490 \mathrm{~nm}$, and emission band slits at $2 \mathrm{~nm}$ ) due to the release kinetics was monitored until the stationary state was reached.

The percentage of peptide-induced dye release $(\% \mathrm{R})$ was calculated from:

$$
\% R=100\left[\left(F_{\mathrm{f}}-F_{\mathrm{i}}\right) /\left(F_{\mathrm{m}}-F_{\mathrm{i}}\right)\right]
$$

where $F_{\mathrm{f}}$ is fluorescence measured in the stationary state (after $1 \mathrm{~h}$ incubation for calcein release and $12.5 \mathrm{~min}$ for release of dextrans), $F_{\mathrm{i}}$ is the initial fluorescence before the addition of the peptides, and $F_{\mathrm{m}}$ is the maximal value after addition of $1 \mathrm{~mm}$ Triton X-100. Spontaneous release of calcein or dextrans was found to be negligible in all cases.

\section{Lipid transbilayer diffusion in LUVs}

Asymmetrically labelled vesicles were prepared as described [39]. The desired amount of 1-lauroyl-2-(1'-pyrenebutyroyl)sn-glycero-3-phosphocholine (py-PtdCho) from a chloroform stock was dried on a glass tube with a nitrogen flux and dissolved in ethanol at the desired concentration. The fluorescent probe in ethanol solution was added to a final concentration of $1 \mu \mathrm{M}$ to buffer A. Then LUVs prepared as described above, but rehydrated in buffer A, were added to a final lipid concentration of $20 \mu \mathrm{M}$. Under these conditions, the labelled lipid is incorporated only into the external 
leaflet of LUVs. Fluorescence emission spectra between 355 and $500 \mathrm{~nm}$ were recorded at room temperature with excitation set at $345 \mathrm{~nm}$, using an LS-50B luminescence spectrometer (Perkin Elmer) and a 1-cm path-length cell with constant stirring. Emission intensities of the excimers $\left(I_{\mathrm{E}}\right.$ at $465 \mathrm{~nm})$ and the monomers $\left(I_{\mathrm{M}}\right.$ at $\left.395 \mathrm{~nm}\right)$ were taken from the spectra to compute $I_{\mathrm{E}} / I_{\mathrm{M}}$ ratios. After incubation for $20 \mathrm{~min}$, to ensure stability, the peptide was added and values of $I_{\mathrm{E}} / I_{\mathrm{M}}$, related to the corresponding emission ratios in the absence of peptides, were plotted against time.

\section{CD spectroscopy}

Samples for CD spectroscopy were prepared at a $30 \mu \mathrm{M}$ concentration of peptide in $10 \mathrm{~mm}$ phosphate buffer at $\mathrm{pH}$ 7. Several percentages of trifluoroethanol, or different concentrations of SDS, below and above the critical micellar concentration, were added to the corresponding samples. Spectra were measured at $20{ }^{\circ} \mathrm{C}$ on a Jasco J-810 CD spectropolarimeter, using a cell of $1 \mathrm{~mm}$ path length. The data were collected every $0.2 \mathrm{~nm}$ at $100 \mathrm{~nm} \cdot \mathrm{min}^{-1}$ from 250 to $185 \mathrm{~nm}$, with a bandwidth of $1 \mathrm{~nm}$, and results were averaged from 10 scans.

Data were analysed with the help of the CDPro software package, which contains three commonly used programs: SELCON3, CONTIN/LL and CDSSTR [42,43]. This software allows the use of different reference sets of proteins, including membrane proteins, increasing the reliability of the analysis.

\section{Acknowledgements}

This work was supported by the Spanish Ministerio de Educación y Ciencia (CTQ2004-03444), the Italian Consiglio Nazionale delle Ricerche (CNR) and the Istituto Trentino di Cultura (ITC). The Spanish Ministerio de Educación y Ciencia is acknowledged for an FPU fellowship (to A.J.G.). We thank Dr Enrique Pérez-Payá for helping with the peptide synthesis and purification. Dr David Vie from the Instituto de Ciencia de los Materiales (Universitat de València) is acknowledged for his assistance with the measurements of vesicle size.

\section{References}

1 Newmeyer DD \& Ferguson-Miller S (2003) Mitochondria: releasing power for life and unleashing the machineries of death. Cell 112, 481-490.

2 Adams JM \& Cory S (1998) The Bcl-2 protein family: arbiters of cell survival. Science 281, 1322-1326.

3 Wei MC, Zong WX, Cheng EH, Lindsten T, Panoutsakopoulou V, Ross AJ, Roth KA, MacGregor GR, Thompson CB \& Korsmeyer SJ (2001) Proapoptotic BAX and BAK: a requisite gateway to mitochondrial dysfunction and death. Science 292, 727-730.
4 Wolter KG, Hsu YT, Smith CL, Nechushtan A, Xi XG \& Youle RJ (1997) Movement of Bax from the cytosol to mitochondria during apoptosis. J Cell Biol 139, 1281-1292.

5 Goping IS, Gross A, Lavoie JN, Nguyen M, Jemmerson R, Roth K, Korsmeyer SJ \& Shore GC (1998) Regulated targeting of BAX to mitochondria. $J$ Cell Biol 143, 207-215.

6 Yethon JA, Epand RF, Leber B, Epand RM \& Andrews DW (2003) Interaction with a membrane surface triggers a reversible conformational change in Bax normally associated with induction of apoptosis. $J$ Biol Chem 278, 48935-48941.

7 Wang K, Yin XM, Chao DT, Milliman CL \& Korsmeyer SJ (1996) BID: a novel BH3 domain-only death agonist. Genes Dev 10, 2859-2869.

8 Desagher S, Osen-Sand A, Nichols A, Eskes R, Montessuit S, Lauper S, Maundrell K, Antonsson B \& Martinou JC (1999) Bid-induced conformational change of Bax is responsible for mitochondrial cytochrome $c$ release during apoptosis. J Cell Biol 144, 891-901.

9 Eskes R, Desagher S, Antonsson B \& Martinou JC (2000) Bid induces the oligomerization and insertion of Bax into the outer mitochondrial membrane. Mol Cell Biol 20, 929-935.

10 Terrones O, Antonsson B, Yamaguchi H, Wang HG, Liu J, Lee RM, Herrmann A \& Basanez G (2004) Lipidic pore formation by the concerted action of proapoptotic BAX and tBID. J Biol Chem 279, 30081-30091.

11 Antonsson B, Montessuit S, Sanchez B \& Martinou JC (2001) Bax is present as a high molecular weight oligomer/complex in the mitochondrial membrane of apoptotic cells. J Biol Chem 276, 11615-11623.

12 Saito M, Korsmeyer SJ \& Schlesinger PH (2000) BAXdependent transport of cytochrome c reconstituted in pure liposomes. Nat Cell Biol 2, 553-555.

13 Antonsson B, Conti F, Ciavatta A, Montessuit S, Lewis S, Martinou I, Bernasconi L, Bernard A, Mermod JJ, Mazzei G, et al. (1997) Inhibition of Bax channel-forming activity by Bcl-2. Science 277, 370-372.

14 Basanez G, Nechushtan A, Drozhinin O, Chanturiya A, Choe E, Tutt S, Wood KA, Hsu Y, Zimmerberg J \& Youle RJ (1999) Bax, but not Bcl-xL, decreases the lifetime of planar phospholipid bilayer membranes at subnanomolar concentrations. Proc Natl Acad Sci USA 96, 5492-5497.

15 Basanez G, Sharpe JC, Galanis J, Brandt TB, Hardwick JM \& Zimmerberg J (2002) Bax-type apoptotic proteins porate pure lipid bilayers through a mechanism sensitive to intrinsic monolayer curvature. J Biol Chem 277, 49360-49365.

16 Sobko AA, Kotova EA, Antonenko YN, Zakharov SD \& Cramer WA (2004) Effect of lipids with different 
spontaneous curvature on the channel activity of colicin E1: evidence in favor of a toroidal pore. FEBS Lett 576, 205-210.

17 Alvarez Valcarcel C, Dalla Serra M, Potrich C, Bernhart I, Tejuca M, Martinez D, Pazos F, Lanio ME \& Menestrina G (2001) Effects of lipid composition on membrane permeabilization by sticholysin I and II, two cytolysins of the sea anemone Stichodactyla helianthus. Biophys J 80, 2761-2774.

18 Anderluh G, Dalla Serra M, Viero G, Guella G, Macek P \& Menestrina G (2003) Pore formation by equinatoxin II, a eukaryotic protein toxin, occurs by induction of nonlamellar lipid structures. J Biol Chem 278, 4521645223.

19 Ludtke SJ, He K, Heller WT, Harroun TA, Yang L \& Huang HW (1996) Membrane pores induced by magainin. Biochemistry 35, 13723-13728.

20 Matsuzaki K, Murase O, Fujii N \& Miyajima K (1996) An antimicrobial peptide, magainin 2, induced rapid flip-flop of phospholipids coupled with pore formation and peptide translocation. Biochemistry 35, 1136111368 .

21 Yang L, Harroun TA, Weiss TM, Ding L \& Huang HW (2001) Barrel-stave model or toroidal model? A case study on melittin pores. Biophys $J$ 81, 1475-1485.

22 Sandre O, Moreaux L \& Brochard-Wyart F (1999) Dynamics of transient pores in stretched vesicles. Proc Natl Acad Sci USA 96, 10591-10596.

23 Fosnaric M, Kralj-Iglic V, Bohinc K, Iglic A \& May S (2003) Stabilization of pores in lipid bilayers by anisotropic inclusions. J Phys Chem B 107, 12519-12526.

24 Muchmore SW, Sattler M, Liang H, Meadows RP, Harlan JE, Yoon HS, Nettesheim D, Chang BS, Thompson CB, Wong SL, et al. (1996) X-ray and NMR structure of human Bcl-xL, an inhibitor of programmed cell death. Nature 381, 335-341.

25 Suzuki M, Youle RJ \& Tjandra N (2000) Structure of bax. Coregulation of dimer formation and intracellular localization. Cell 103, 645-654.

26 Garcia-Saez AJ, Mingarro I, Perez-Paya E \& Salgado J (2004) Membrane-Insertion Fragments of Bcl-x (L), Bax, and Bid. Biochemistry 43, 10930-10943.

27 Heimlich G, McKinnon AD, Bernardo K, Brdiczka D, Reed JC, Kain R, Kronke M \& Jurgensmeier JM (2004) Bax-induced cytochrome c release from mitochondria depends on alpha-helices-5 and -6. Biochem $J$ 378, 247-255.

28 Garcia-Saez AJ, Coraiola M, Dalla Serra M, Mingarro I, Menestrina G \& Salgado J (2005) Peptides derived from apoptotic bax and bid reproduce the poration activity of the parent full-length proteins. Biophys $J \mathbf{8 8}$, 3976-3990.

29 Allende D, Simon SA \& McIntosh TJ (2005) Melittininduced bilayer leakage depends on lipid material properties: evidence for toroidal pores. Biophys $J \mathbf{8 8}$, 1828-1837.

30 Matsuzaki K, Sugishita K, Ishibe N, Ueha M, Nakata S, Miyajima K \& Epand RM (1998)

Relationship of membrane curvature to the formation of pores by magainin 2. Biochemistry 37, 1185611863.

31 Puech PH, Borghi N, Karatekin E \& Brochard-Wyart F (2003) Line thermodynamics: adsorption at a membrane edge. Phys Rev Lett 90, 128304.

32 Karatekin E, Sandre O, Guitouni H, Borghi N, Puech PH \& Brochard-Wyart F (2003) Cascades of transient pores in giant vesicles: line tension and transport. Biophys $J$ 84, 1734-1749.

33 de Kruijff B \& Cullis PR (1980) The influence of poly (1-lysine) on phospholipid polymorphism. Evidence that electrostatic polypeptide-phospholipid interactions can modulate bilayer/non-bilayer transitions. Biochim Biophys Acta 601, 235-240.

34 Benachir T \& Lafleur M (1995) Study of vesicle leakage induced by melittin. Biochim Biophys Acta 1235, 452-460.

35 Kuwana T, Mackey MR, Perkins G, Ellisman MH, Latterich M, Schneiter R, Green DR \& Newmeyer DD (2002) Bid, bax, and lipids cooperate to form supramolecular openings in the outer mitochondrial membrane. Cell 111, 331-342.

36 Dufourcq J, Faucon JF, Fourche G, Dasseux JL, Le Maire M \& Gulik-Krzywicki T (1986) Morphological changes of phosphatidylcholine bilayers induced by melittin: vesicularization, fusion, discoidal particles. Biochim Biophys Acta 859, 33-48.

37 Amsden B (2002) Modeling solute diffusion in aqueous polymer solutions. Polymer 43, 1623-1630.

38 Epand RF, Martinou JC, Montessuit S \& Epand RM (2003) Transbilayer lipid diffusion promoted by Bax: implications for apoptosis. Biochemistry 42, 1457614582.

39 Muller P, Schiller S, Wieprecht T, Dathe M \& Herrmann A (2000) Continuous measurement of rapid transbilayer movement of a pyrene-labeled phospholipid analogue. Chem Phys Lipids 106, 89-99.

40 Dalla Serra M \& Menestrina G (2003) Liposomes in the study of pore-forming toxins. Methods Enzymol 372, 99-124.

41 Santos NC \& Castanho MA (1996) Teaching light scattering spectroscopy: the dimension and shape of tobacco mosaic virus. Biophys $J$ 71, 1641-1650.

42 Sreerama N \& Woody RW (2000) Estimation of protein secondary structure from circular dichroism spectra: comparison of CONTIN, SELCON, and CDSSTR methods with an expanded reference set. Anal Biochem 287, 252-260.

43 Sreerama N \& Woody RW (2004) On the analysis of membrane protein circular dichroism spectra. Protein Sci 13, 100-112. 